\title{
Production and collections of antiprotons
}

\author{
V. Lebedev
}

\section{Antiproton yield from the target}

The simulations of antiproton production in nickel target were performed by P. Bussey with MARS code developed by N. Mokhov. Figure 1 presents the total yield of antiprotons produced by $120 \mathrm{GeV}$ proton beam into momentum acceptance of $\pm 2.25 \%$ around $8 \mathrm{GeV}$ kinetic energy as function of the target length. One can see that the total yield grows fast with target length. In reality only fraction of antiprotons can be accepted into the debuncher and one needs to find optimum conditions to inject maximum antiprotons into a finite phase space of the ring. Figure 2 shows coordinates of antiprotons in $x-x^{\prime}$ phase space produced by the proton beam with rms beam size of $100 \mu \mathrm{m}$ in the $8 \mathrm{~cm}$ long nickel target. Particle $x$-coordinates were translated to the longitudinal coordinate at which the second order moments $\left\langle x \theta_{x}\right\rangle$ and $\left\langle y \theta_{y}\right\rangle$ are equal to zero. If there would not be scattering and absorption of antiprotons in the target this coordinate (waist position) would be in the center of the target. In reality it is shifted downstream of the target center. We denote this position by $\delta s$. For $8 \mathrm{~cm}$ target it is equal to about $2.1 \mathrm{~mm}$. As one can see the total angular spread is about $100 \mathrm{mrad}$. The circle on the plot presents the boundary of phase space with acceptance $\varepsilon=25 \mathrm{~mm}$ mrad and beta-function $\beta^{*}=1.5 \mathrm{~cm}$.

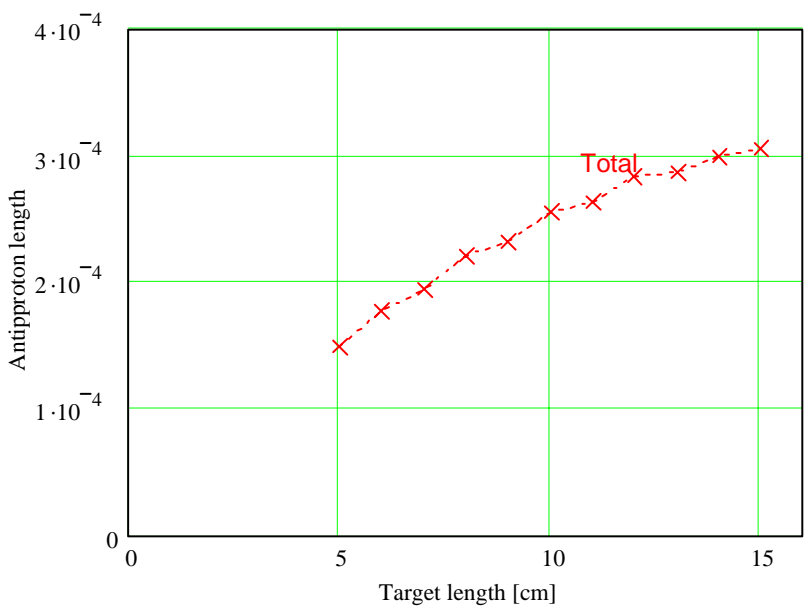

Figure 1. Dependence of total antiproton yield on the length of nickel target for proton beam of $120 \mathrm{GeV}$; momentum acceptance is $\pm 2.25 \%$.

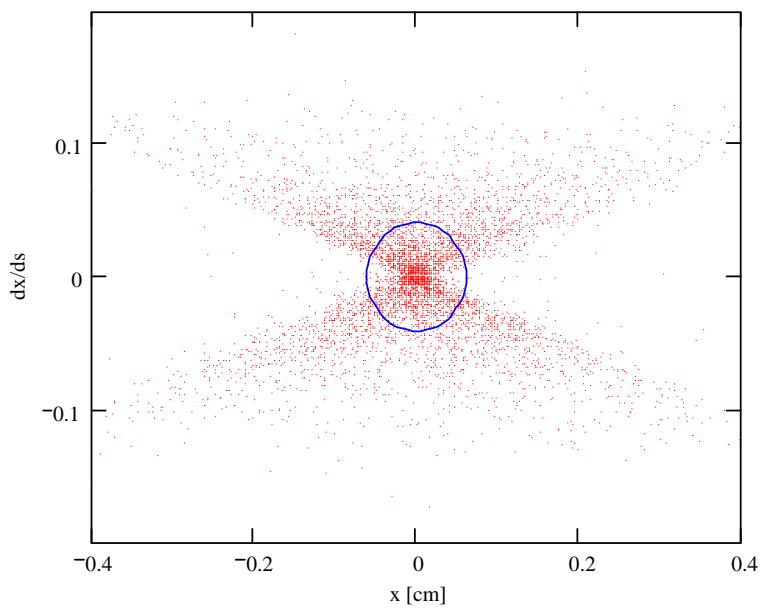

Figure 2. Coordinates of antiprotons in $x-x^{\prime}$ phase space simulated with MARS code for proton beam of $120 \mathrm{GeV}$ and rms beam size of $100 \mu \mathrm{m}$. The circle inscribes phase space with $\varepsilon=25 \mathrm{~mm} \mathrm{mrad}$ and $\beta^{*}=1.5 \mathrm{~cm}$. 

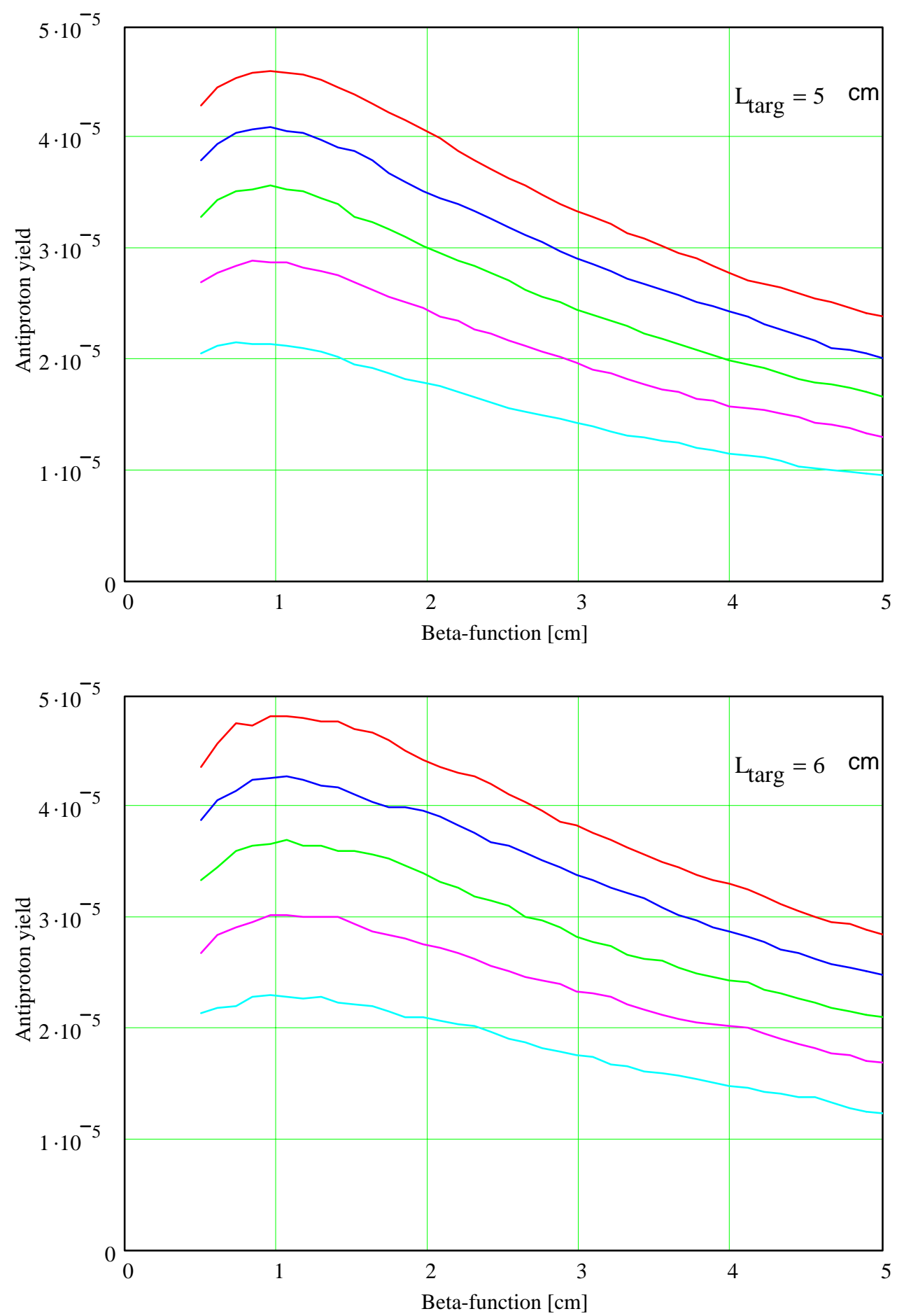

Figure 3. Dependence of antiproton yield into $\pm 2.25 \%$ momentum spread on the beta-function at the target for the beam acceptances of 15,20,25, 30 and $35 \mathrm{~mm} \mathrm{mrad}$, and target length of 5 and $6 \mathrm{~cm}$; waist positions are 0.5 and $1.55 \mathrm{~mm}$ for target lengths 5 and $6 \mathrm{~cm}$, correspondingly. Proton beam energy is 120 $\mathrm{GeV}$, and rms beam size at the target is $100 \mu \mathrm{m}$. Kinetic energy of antiprotons is $8 \mathrm{GeV}$. 
PBAR Note 666
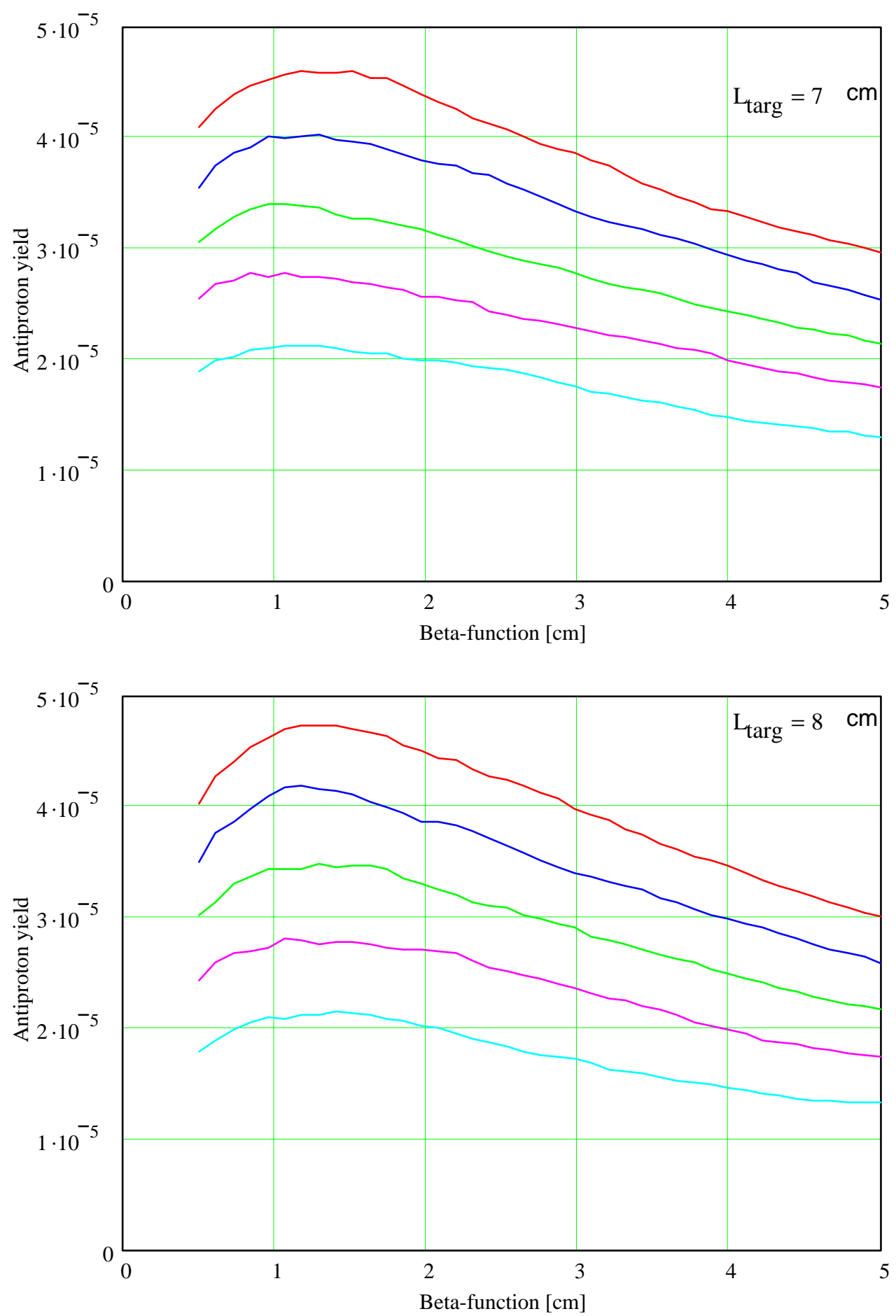

Figure 4. Dependence of antiproton yield into $\pm 2.25 \%$ momentum spread on the beta-function at the target for the beam acceptances of 15,20,25, 30 and $35 \mathrm{~mm} \mathrm{mrad}$, and target lengths of 7 and $8 \mathrm{~cm}$; waist positions are 2.3 and $2.15 \mathrm{~mm}$ for target lengths 7 and $8 \mathrm{~cm}$, correspondingly. Proton beam energy is 120 $\mathrm{GeV}$, and rms beam size at the target is $100 \mu \mathrm{m}$. Kinetic energy of antiprotons is $8 \mathrm{GeV}$. 
PBAR Note 666
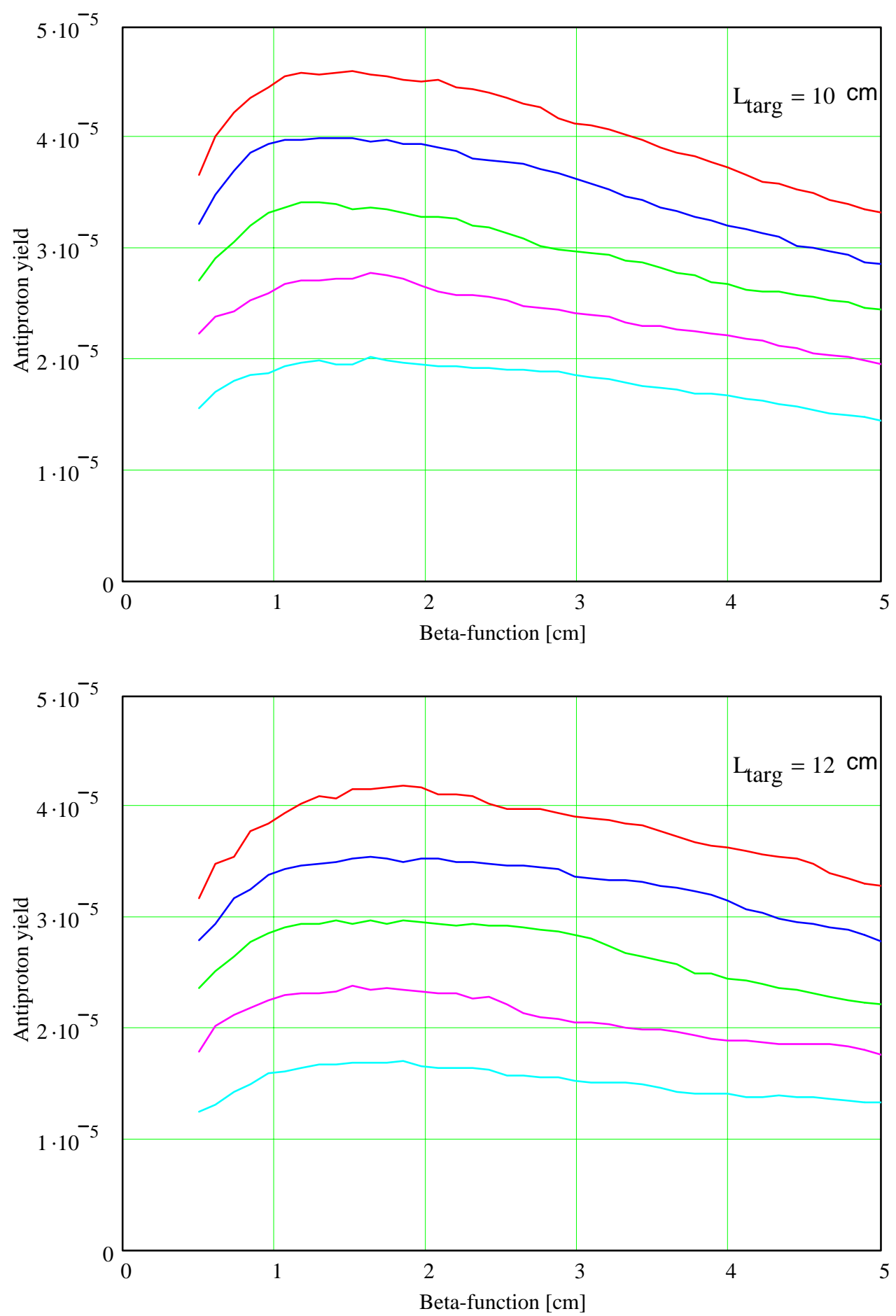

Figure 5. Dependence of antiproton yield into $\pm 2.25 \%$ momentum spread on the beta-function at the target for the beam acceptances of 15,20,25, 30 and $35 \mathrm{~mm} \mathrm{mrad}$, and target length of 10 and $12 \mathrm{~cm}$; waist positions are 4.2 and $5.9 \mathrm{~mm}$ for target lengths 10 and $12 \mathrm{~cm}$, correspondingly. Proton beam energy is 120 $\mathrm{GeV}$, and rms beam size at the target is $100 \mu \mathrm{m}$. Kinetic energy of antiprotons is $8 \mathrm{GeV}$. 

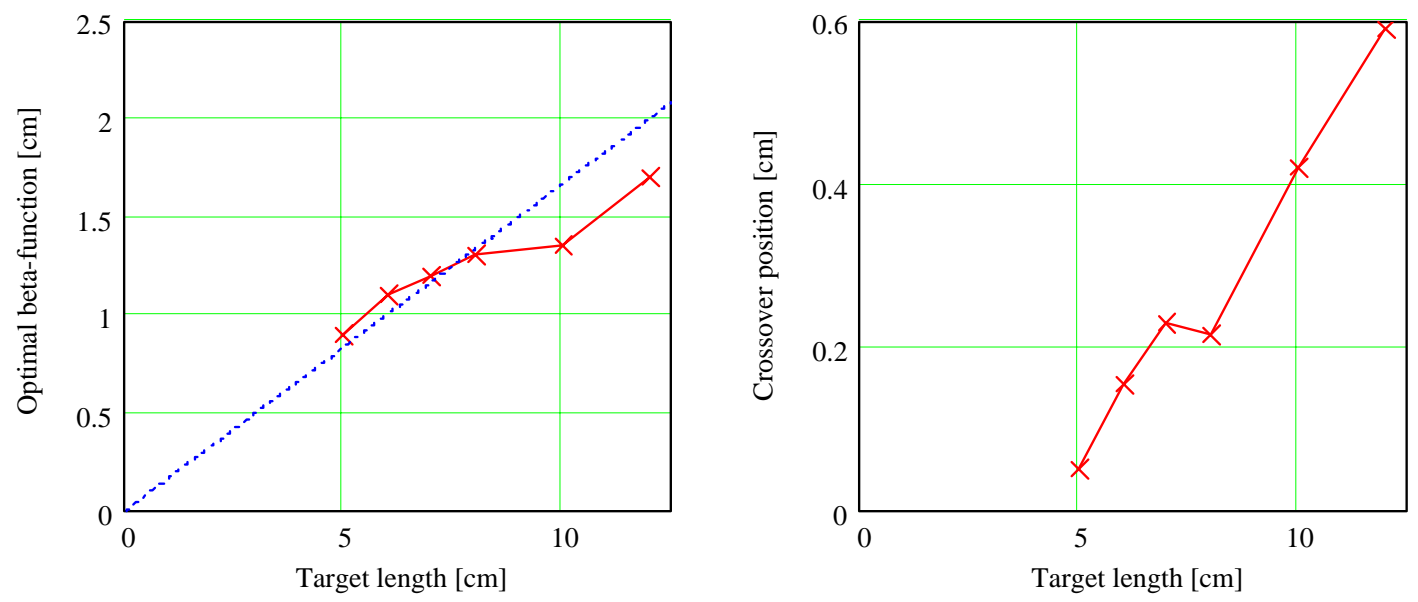

Figure 6. Dependence of the optimal beta-function and the waist position on the target length. Proton beam energy is $120 \mathrm{GeV}$, and rms beam size at the target is $100 \mu \mathrm{m}$.

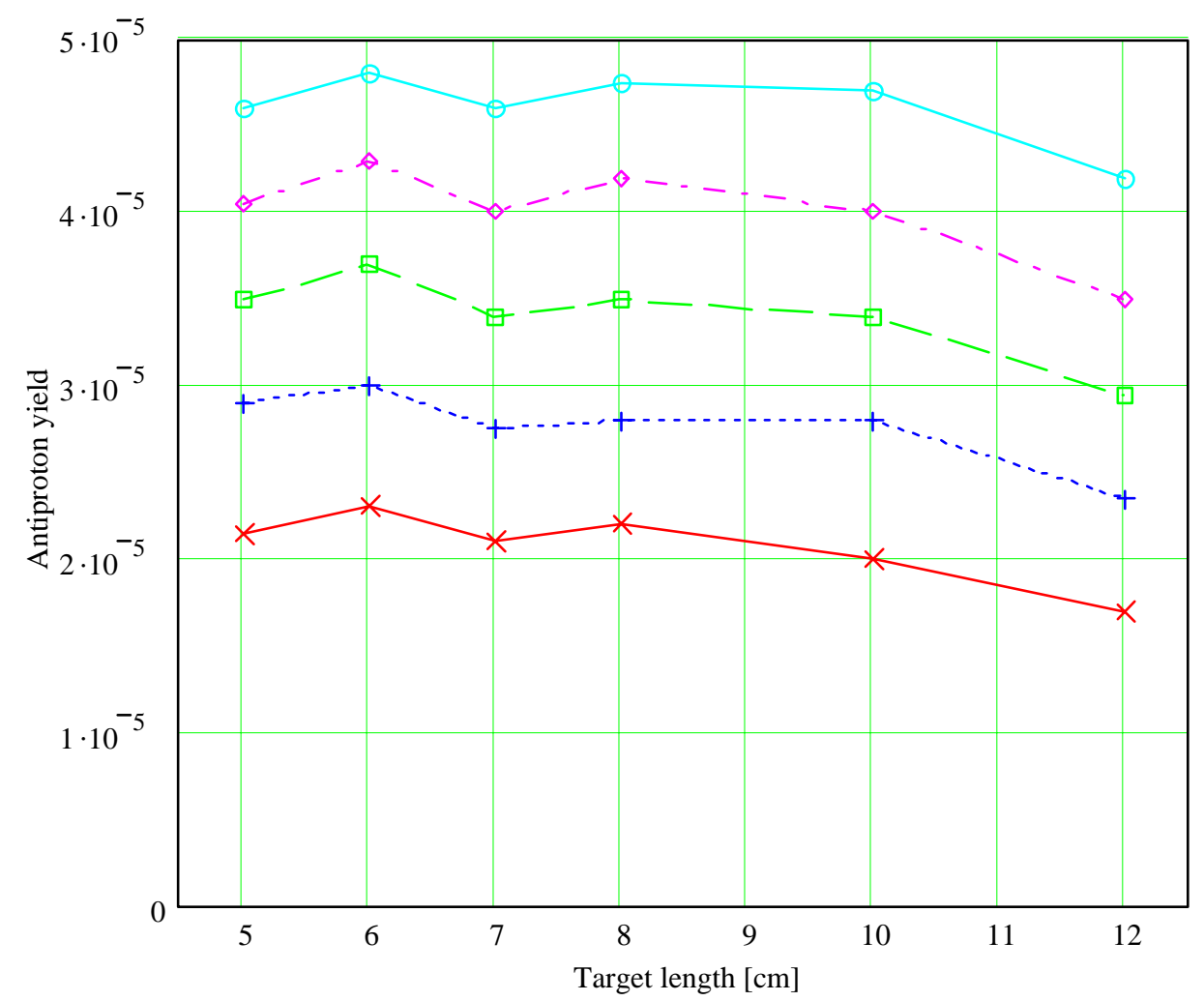

Figure 7. Dependence of maximum antiproton yield into $\pm 2.25 \%$ momentum spread on the target length for the beam acceptances of $15,20,25,30$ and $35 \mathrm{~mm}$ mrad. Proton beam energy is $120 \mathrm{GeV}$, and rms beam size at the target is $100 \mu \mathrm{m}$. Kinetic energy of antiprotons is $8 \mathrm{GeV}$. 
Figures 3-5 show the antiproton yield as function of $\beta^{*}$ for different target lengths and machine acceptances. Horizontal and vertical acceptances are considered to be equal; and the momentum acceptance is equal $\pm 2.25 \%$. Thus, antiprotons within the phase space determined by the following equation,

$$
\left(\frac{x_{i}^{2}}{\beta^{*}}+x_{i}^{\prime 2} \beta^{*}\right)+\left(\frac{y_{i}^{2}}{\beta^{*}}+y_{i}^{\prime 2} \beta^{*}\right) \leq \varepsilon \quad,\left|\frac{\Delta p_{i}}{p_{0}}\right| \leq 0.0225,
$$

were considered to be accepted to the ring. For given target length the maximum yield is achieved at optimal beta-function. As one can see in Figures 3-5 for given target length this optimal beta-function is approximately the same for different machine acceptances. Figure 6 shows the dependence of optimal beta-function and the waist position on the target length. The waist displacement from the target center is related to scattering and absorption of antiprotons in the target. The optimum beta-function is approximately $1 / 6$ of the target length.

Figure 7 presents the dependence of maximum antiproton yield (at optimal betafunction) on target length for different machine acceptances. One can see that the optimal target length is about $6 \mathrm{~cm}$. There is no significant decrease in the yield for slightly longer targets but the optimum beta-function grows with target length allowing to use a weaker lithium lens for antiproton collection. That can be profitable in optimizing the design.

\section{Scattering and absorption of antiprotons in lithium lens}

Nuclear scattering and absorption of antiprotons in the lithium lens is the major mechanism for antiproton loss in the lens. The loss of antiprotons due to their strong interactions with lens material can be estimated by the following expression,

$$
\kappa_{\text {lens }}=\exp \left(-\frac{L_{L i}}{L_{A b s_{L i}}}-\frac{L_{B e}}{L_{A b s_{B e}}}\right) \approx 0.82,
$$

where $L_{L i}=15.5 \mathrm{~cm}$ and $L_{B e}=1.2$ $\mathrm{cm}$ are total lengths of lithium and beryllium crossed by the beam, and $L_{A b s_{L i}}=102 \mathrm{~cm}$ and $L_{A b s_{B e}}=30.2 \mathrm{~cm}$ are nuclear collision lengths for lithium and beryllium. This estimate is in a remarkable coincidence with results of MARS simulations.

Multiple scattering in the lens can be estimated by the following formula,

$$
\begin{aligned}
\sqrt{\overline{\theta^{2}}}= & \frac{13.6 \mathrm{MeV}}{\beta P c} \sqrt{\frac{L_{L i}}{X_{L i}}+\frac{L_{B e}}{X_{B e}}} \\
& =0.635 \mathrm{mrad},
\end{aligned}
$$

where $X_{L i}=155 \mathrm{~cm}$ and $X_{B e}=35.3$

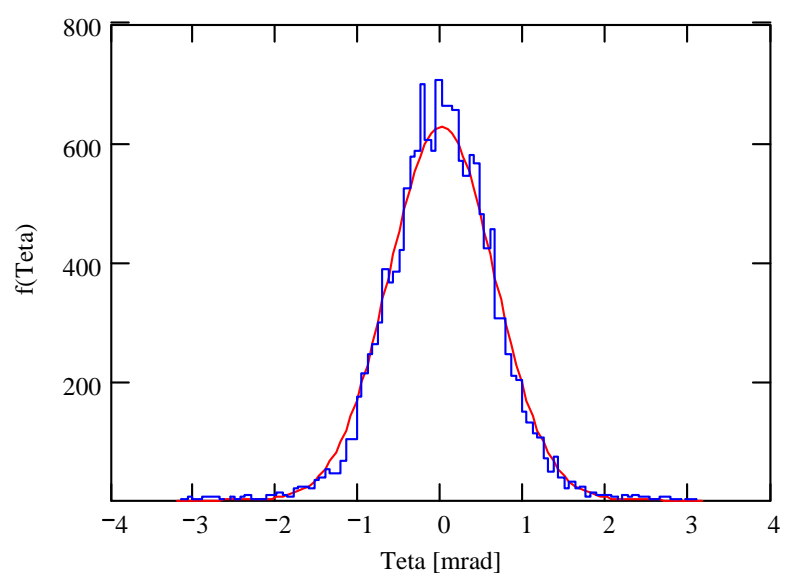

Figure 8. Distribution functions of point like beam after passing through lithium lens simulated by MARS and computed with use of multiple scattering formula of Eq.

$\mathrm{cm}$ are the radiation lengths for 
lithium and beryllium. Figure 8 presents a comparison of results obtained with Eq. (3) and results of MARS simulations. There is good coincidence between simulation and Eq.(3) for angles below 2 mrad. For large angles, as it is expected, MARS produces long non-gaussian tails but only small fraction of the particles are there. As will be seen below multiple scattering in the lens is sufficiently small and does not significantly affect the antiproton yield. That leaves the nuclear absorption as major mechanism for particle loss in the lens with about $\% 1$ loss for every centimeter of lithium.

\section{Antiproton yield in approximation of lithium lens linear focusing}

For given acceptance and beta-function on the target the distance between the target and the lens and the lens current are chosen so that the maximum beam size in the lens would be equal to its radius and the beam size in the first triplet of AP2 line would be minimized. Figures 9 and 10 present the beam sizes and beta-functions optimized this way for beam acceptances of 15 and $25 \mathrm{~mm}$ mrad. One can see that such optimization changes beta-functions in the triplet. To avoid an envelope mismatch at injection into the debuncher this beta-function change has to be corrected using other quads of AP2 line.
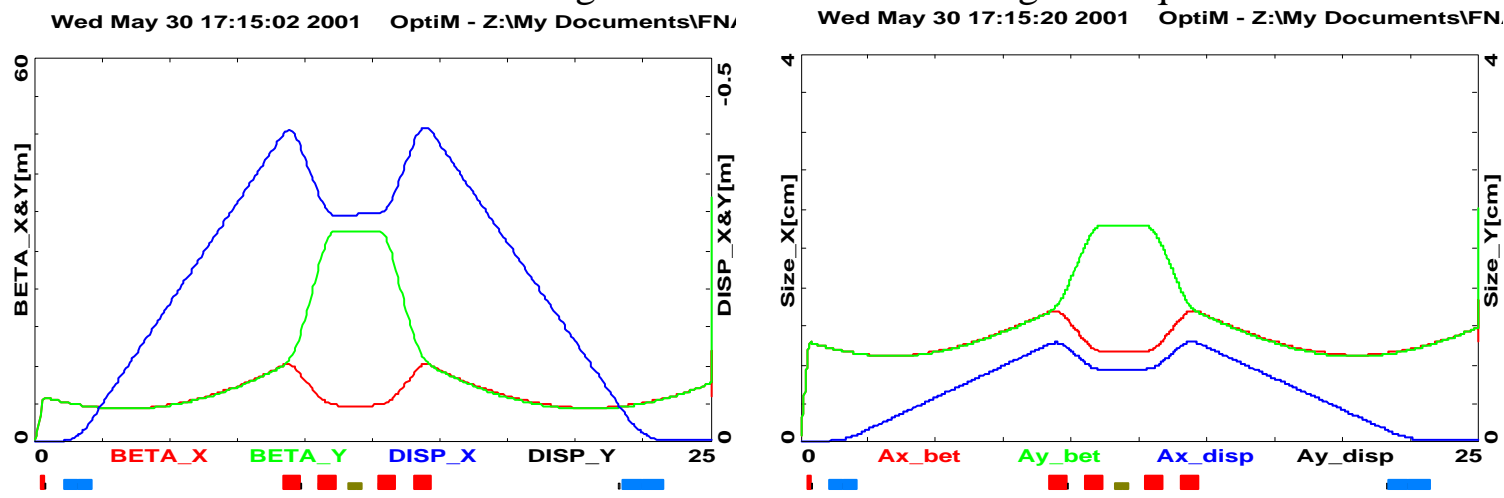

Figure 9. Dispersion beta-functions (left) and beam envelopes (right) at the beginning of AP2 line for $\varepsilon=15$ $\mathrm{mm} \cdot \mathrm{mrad}$ and $\beta=1.38 \mathrm{~cm}$. Lithium lens gradient is $72 \mathrm{kG} / \mathrm{cm}$.

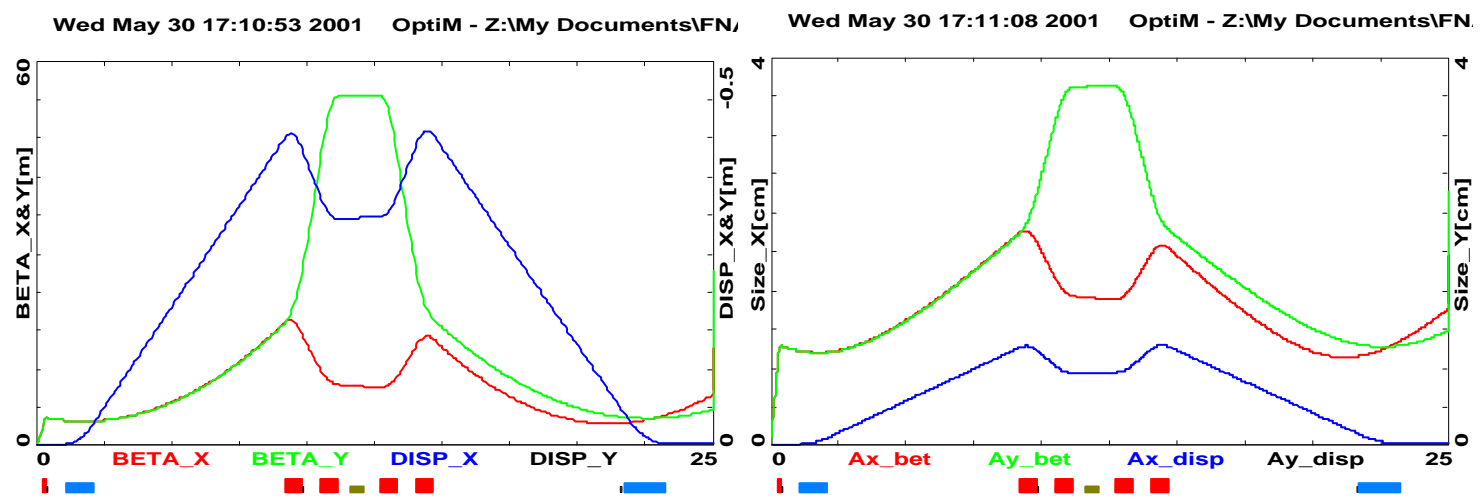

Figure 10. Dispersion beta-functions (left) and beam envelopes (right) at the beginning of AP2 line for $\varepsilon=25 \mathrm{~mm} \cdot \mathrm{mrad}$ and $\beta^{*}=2.3 \mathrm{~cm}$. Lithium lens gradient is $72 \mathrm{kG} / \mathrm{cm}$.

Current design of the lithium lens limits its gradient to about $75 \mathrm{kG} / \mathrm{cm}$. Previous measurements of antiproton collection exhibited fast growth of the antiproton yield with increasing lens strength. Therefore we need to investigate possible lens redesigns and related to it benefits. The simplest possible lens modification is an increase of lithium lens length from 15 to $18 \mathrm{~cm}$, which increases the lens focusing strength by $20 \%$ but also 
increases the nuclear absorption from $18 \%$ to $20.3 \%$. Figure 11 presents dependence of antiproton yield as function of the lens gradient into acceptances of 15, 25 and $35 \mathrm{~mm}$ mrad. Other parameters such as the target length, the beta-function at target center and the lens-to-target distance were optimized to get the maximum yield. In comparison with Figures 1, 3-5 and 7 the nuclear absorption in the lens is taken into account here. As will be seen below the non-linearity of lens focusing does not deteriorate the antiproton yield and therefore the yield presented in Figure 11 should be close to the ultimate yield achievable in the experiment. As one can see the current lens is sufficiently strong for $15 \mathrm{~mm} \mathrm{mrad}$ acceptance. For 25 and $35 \mathrm{~mm} \mathrm{mrad}$ acceptances we lose about $13 \%$ and $16 \%$ in antiproton yield in comparison with optimal $15 \mathrm{~cm}$ lens. For both 25 and 35 $\mathrm{mm} \mathrm{mrad}$ an increase of lithium lens length from 15 to $18 \mathrm{~cm}$ will allow gaining back about 5\% or, without change of antiproton yield, reducing lens gradient to $68 \mathrm{kG} / \mathrm{cm}$, which is expected to increase significantly the lens lifetime. Figure 12 presents the lens gradient and the target-to-lens distance (center-tocenter) optimized to reach maximum antiproton yield for $25 \mathrm{~mm} \mathrm{mrad}$ acceptance. Note that at $35 \mathrm{~mm}$ mrad a lens with $1 \mathrm{~cm}$ lens radius is barely sufficient to avoid scraping in the first triplet. Further increase of the acceptance requires an increase of lithium lens radius (preferable) or an aperture increase in the first triplet.

Figure 13 presents the dependence of antiproton yield on the proton beam size for the acceptance of $25 \mathrm{~mm} \mathrm{mrad}$, target length of $8 \mathrm{~cm}$ and different beta-functions on the target. One can see that the antiproton yield begins to decrease at beam sizes above about $130 \mu \mathrm{m}$. There is no severe optics limitations preventing the proton beam size to be focused into this or even smaller size, but there is a danger of destroying the target by the beam due to shock waves and target
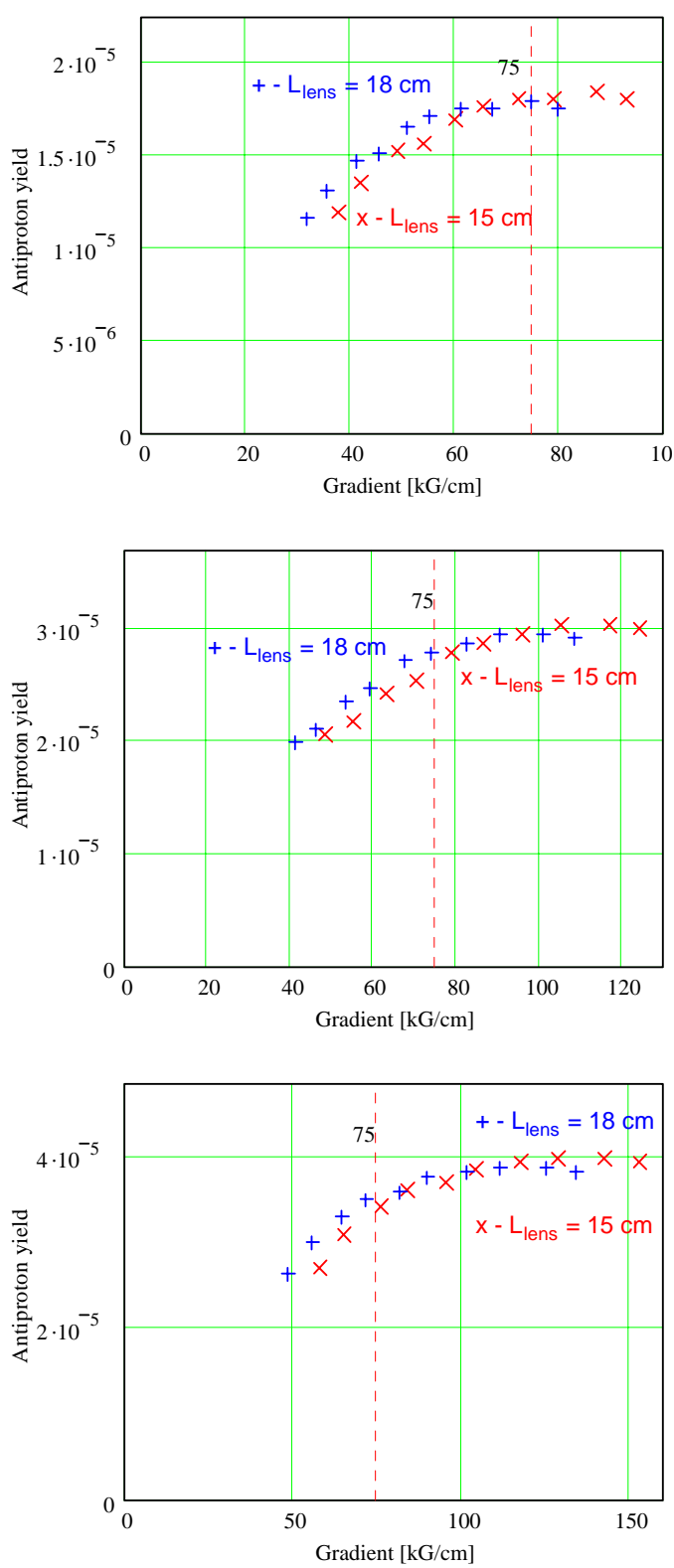

Figure 11. Dependence of optimized antiproton yield on lithium lens gradient for acceptances of $15 \mathrm{~mm} \mathrm{mrad}$ (top), $25 \mathrm{~mm}$ mrad (middle) and 35 $\mathrm{mm}$ mrad (bottom); $\times$ - lens length of $15 \mathrm{~cm},+-$ lens length of $18 \mathrm{~cm}$; lens radius is $1 \mathrm{~cm}$. Vertical dotted line shows current limit for the lens gradient of $75 \mathrm{kG} / \mathrm{cm}$. 
melting. The nickel target can sustain about $900 \mathrm{~J} / \mathrm{g}$ energy deposition. This energy
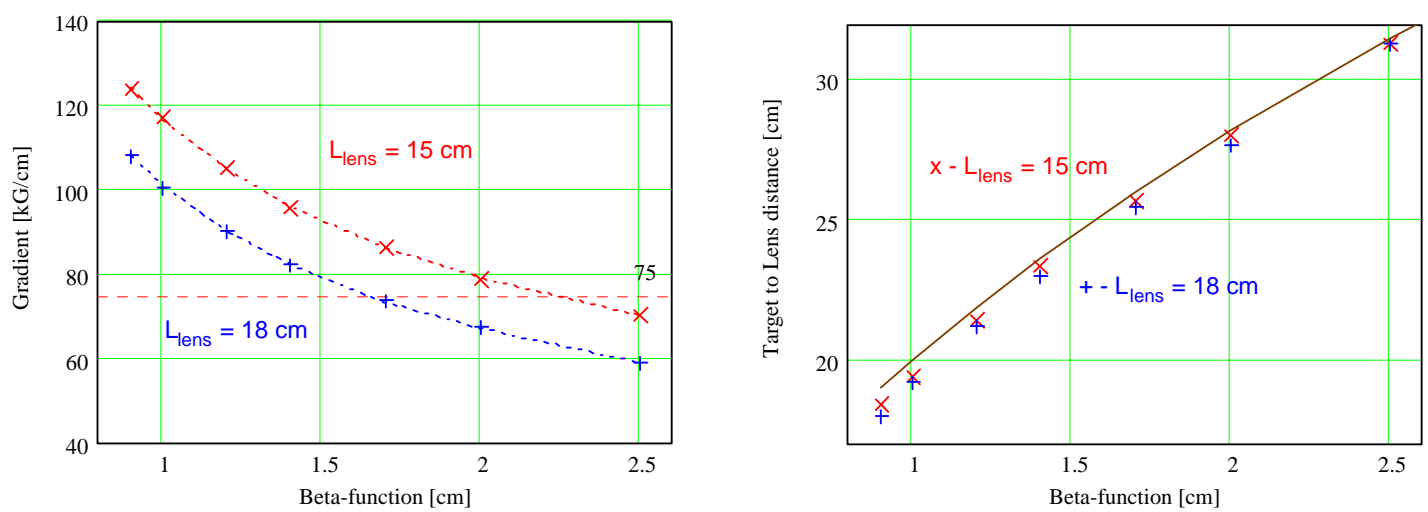

Figure 12. The lens gradient (left) and the center-to-center target-to-lens distance (right) optimized for beam acceptance of $25 \mathrm{~mm}$ mrad.

deposition grows fast with increase of proton beam intensity and decreases the proton beam size. If we would not like to loose antiproton yield due to oversized proton beam we need to keep the rms beam size at $130 \mu \mathrm{m}$. Then the energy deposition limits the number of antiprotons to about $3 \cdot 10^{12}$. Further increase of the proton beam intensity requires an increase of the beam size or its swiping on the target.

\section{Nonlinearity of lithium lens focusing}

Major non-linearity in the lithium lens focusing is related to the skin effect. The lens current represents half period sinusoidal pulse $350 \mu$ s long. The skin depth for frequency $1 /(2 * 0.00035) \approx 1400$

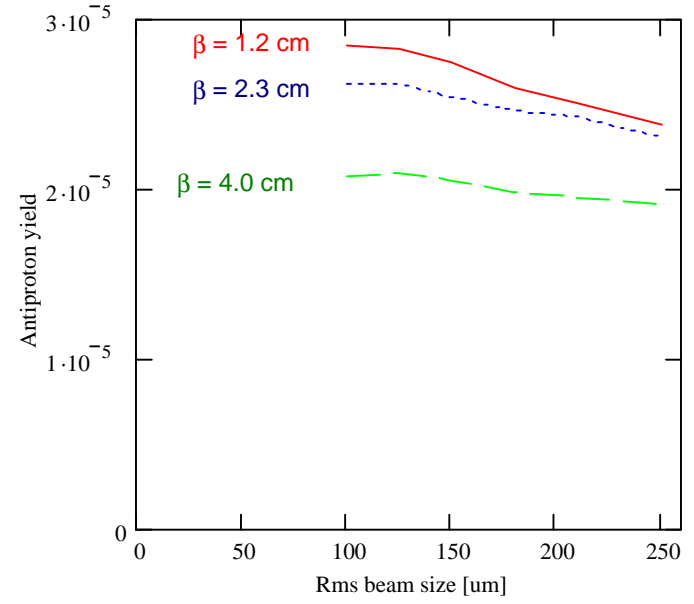

Figure 13. Dependence of antiproton yield on rms size of the proton beam for acceptance of $25 \mathrm{~mm}$ $\mathrm{mrad}$, target length of $8 \mathrm{~cm}$ and beta-function on the target equal to $1.2 \mathrm{~cm}$ - solid curve, $2.3 \mathrm{~cm}$ - dotted curve, and $4 \mathrm{~cm}$ - dashed curve. Antiproton absorption in the lens is taken into account. $\mathrm{Hz}$ is $4.5 \mathrm{~mm}$. That is twice smaller than the lens radius and implies that there is significant delay in penetration of magnetic field in to the lens. Figure 14 shows results of calculations of magnetic field penetration into lithium cylinder with $1 \mathrm{~cm}$ radius. It was obtained by expending the pulse into Fourier series, finding solution for harmonics and performing inverse Fourier transform numerically. One can see that the maximum gradient is achieved at RF phases between 30 and $60 \mathrm{deg}$. There is also a solution for continuous sinusoidal wave shown in the figure. Although this solution is very different at the beginning of the pulse it comes closer later and there is a negligible difference for the 30 to $60 \mathrm{deg}$. phases of interest. Therefore we will use this solution, 

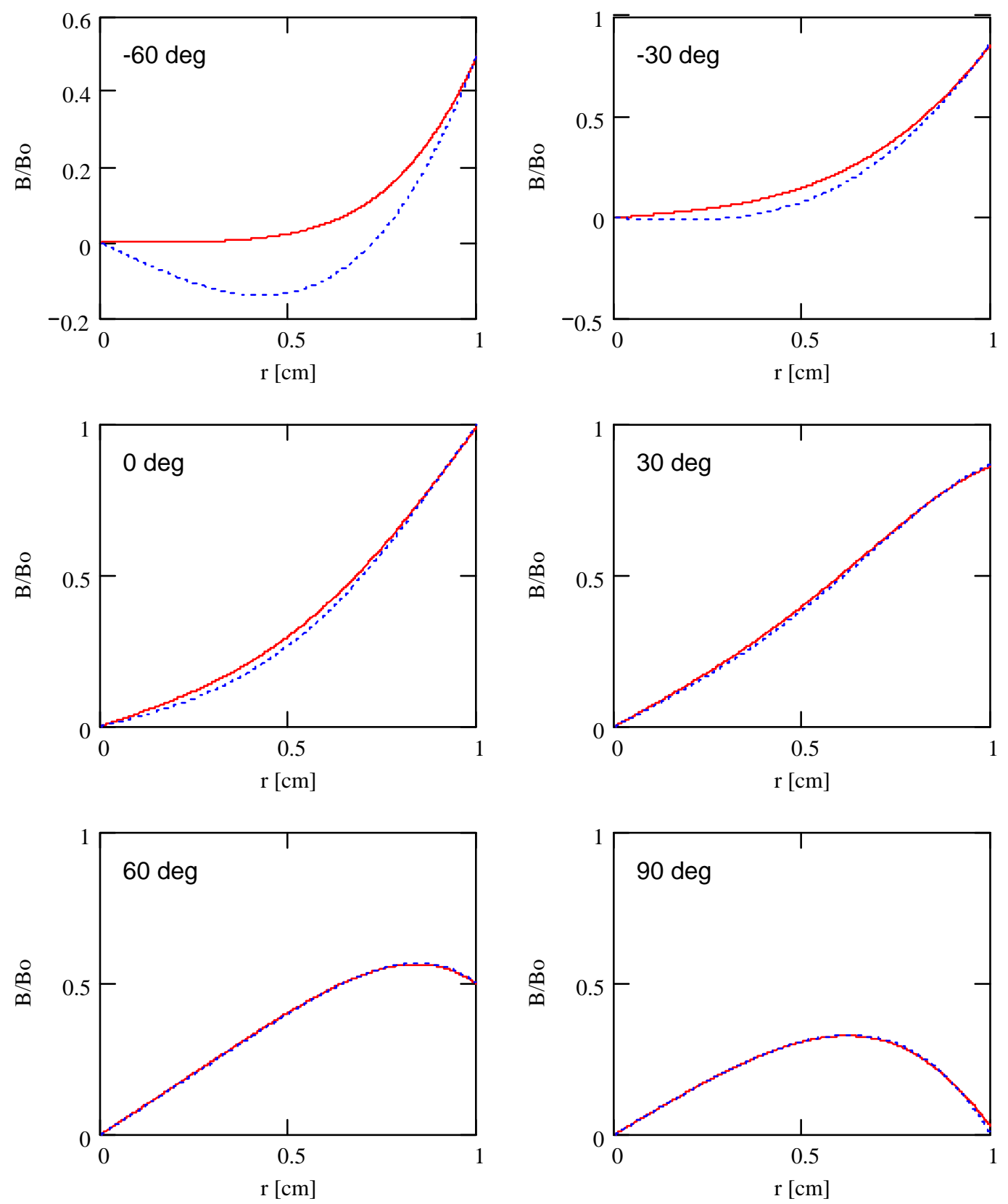

Figure 14. Calculated dependence of lens magnetic field on radius for different times during half period sinusoidal pulse of $350 \mu \mathrm{s}$ long. Time is expressed through the RF phase so that the end and the beginning of the pulse correspond to $\pm 90 \mathrm{deg}$. Dotted line represents solution for continuous sinusoidal wave.

$$
B(r, t)=\frac{2 I_{0}}{c r_{0}} \operatorname{Re}\left(\frac{\operatorname{ber}_{1}(\sqrt{2} r / \delta)+i \operatorname{bei}_{1}(\sqrt{2} r / \delta)}{\operatorname{ber}_{1}\left(\sqrt{2} r_{0} / \delta\right)+i \operatorname{bei}_{1}\left(\sqrt{2} r_{0} / \delta\right)} e^{i \omega t}\right),
$$

for further calculations. Here $\delta$ is the skin-depth for frequency $f=1 /(2 T), T$ is the duration of the pulse, $I_{0}$ is the current amplitude, $r_{0}$ is the radius of lithium cylinder, and 
$\operatorname{ber}(x)$ and $\operatorname{bei}(x)$ are the modified Bessel functions. Expending Bessel functions into Fourier series

$$
\begin{aligned}
& \operatorname{ber}_{1}(x) \approx f_{r}(x) \equiv \frac{1}{\sqrt{2}}\left(-\frac{x}{2}-\frac{x^{3}}{16}+\frac{x^{5}}{384}+\frac{x^{7}}{18432}-\frac{x^{9}}{1474560}\right), \\
& \operatorname{bei}_{1}(x) \approx f_{i}(x) \equiv \frac{1}{\sqrt{2}}\left(\frac{x}{2}-\frac{x^{3}}{16}-\frac{x^{5}}{384}+\frac{x^{7}}{18432}+\frac{x^{9}}{1474560}\right),
\end{aligned}
$$

we finally obtain the expression

$$
B(r, t)=\frac{2 I_{0}}{c r_{0}} \frac{\left(\frac{x}{2}-\frac{x^{5}}{384}+\frac{x^{9}}{147456}\right) \cos \tilde{\psi}-\left(\frac{x^{3}}{16}-\frac{x^{7}}{18432}\right) \sin \tilde{\psi}}{\sqrt{f_{r}^{2}\left(x_{0}\right)+f_{r}^{2}\left(x_{0}\right)}},
$$

which approximate the solution with sufficient accuracy. Here

$$
\tilde{\psi}=\omega t-\psi \quad, \quad \psi=\operatorname{atan}\left(\frac{f_{i}\left(x_{0}\right)}{f_{r}\left(x_{0}\right)}\right)+\frac{\pi}{4} \quad, \quad x=\sqrt{2} \frac{r}{\delta} \quad, \quad x_{0}=\sqrt{2} \frac{r_{0}}{\delta} \quad .
$$

For Fermilab lithium lens parameters $\psi=66.5$ deg. This phase corresponds to RF phase when maximum magnetic field gradient is achieved in the center of lens. Maximum linearity of the gradient is achieved at about $45 \mathrm{deg}$ as shown in Figure 15. Gradient variations across the lens cross-section achieve $\pm 7 \%$. The mean value of the gradient is about $77 \%$ of the gradient calculated without skin-effect taken into account.

Temperature gradient across the lithium cylinder causes additional non-linearity of the lens focusing. The gradient is related to the lens heating by the current pulses. For $1.5 \mathrm{~s}$ repetition time the average power left in the lens is about $100 \mathrm{~W} / \mathrm{cm}$. It produces a temperature gradient across the lens so that the exterior have lower temperature and, consequently, lower resistivity. It produces higher current density in the exterior, which is

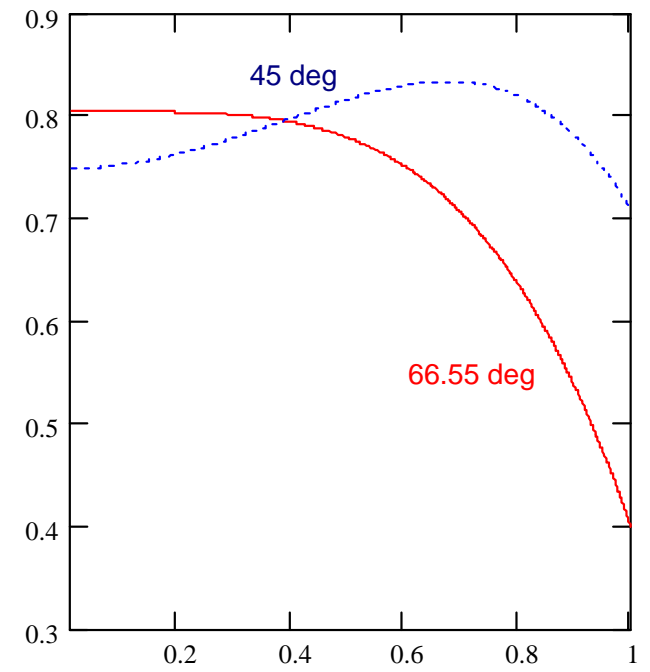

Figure 15. Calculated dependence of lens magnetic field on radius for time when the maximum linearity of the focusing ( $45 \mathrm{deg}$ ) and the maximum gradient $(66.55 \mathrm{deg})$ in the center are achieved. partially compensating magnetic field nonlinearity due to the skin effect. The worst-case estimate can be done for a stationary case. Then, the temperature dependence on radius is:

$$
T(r)=T(0)+\frac{P}{4 \pi \kappa} \frac{r^{2}}{r_{0}^{2}},
$$

where $\kappa=0.82 \mathrm{~W} / \mathrm{cm} / \mathrm{K}$ is thermal conductivity of lithium, and $P$ is power per unit length. For $P=110 \mathrm{~W} / \mathrm{cm}$ one obtain the temperature difference of $10 \mathrm{~K}$ and the 
corresponding current density change, $\Delta j / j$, of about $4 \%$. That yields $2 \%$ correction for magnetic field with dependence on radius described by the following formula:

$$
B(r)=B_{0} \frac{r}{r_{0}}\left(1+\frac{1}{2} \frac{\Delta j}{j} \frac{r^{2}}{r_{0}^{2}}\right) .
$$

In reality the time between pulses is longer than the decay time of temperature wave,

$$
\tau_{Q}=\frac{C r_{0}^{2}}{2 \kappa} \approx 1.2 \mathrm{~s},
$$

where $C=1.95 \mathrm{~J} / \mathrm{K} / \mathrm{cm}^{3}$ is the heat capacity of lithium. That determines that the actual temperature difference is well below the above estimate. Note that the sign of this nonlinearity is opposite to the sign of non-linearity due to skin effect and, consequently, it partially compensates the skin effect non-linearity.

Non-linearities due to the lens edges are even smaller than due to temperature gradient. For the stationary case in the lens body we can expend the current density from the lens axis,

$$
j_{z}(r, z)=\left.j_{z}(z)\right|_{r=0}-\frac{r^{2}}{4} \frac{d^{2}}{d z^{2}}\left(\left.j_{z}(z)\right|_{r=0}\right)+\frac{r^{4}}{64} \frac{d^{4}}{d z^{4}}\left(\left.j_{z}(z)\right|_{r=0}\right)+\ldots .
$$

That yields the following expansion for magnetic field,

$$
B_{\theta}(r, z)=\frac{2 \pi}{c}\left[\left.r j_{z}(z)\right|_{r=0}-\frac{r^{3}}{8} \frac{d^{2}}{d z^{2}}\left(\left.j_{z}(z)\right|_{r=0}\right)+\frac{r^{5}}{192} \frac{d^{4}}{d z^{4}}\left(\left.j_{z}(z)\right|_{r=0}\right)\right]+\ldots .
$$

Integrating it with the equation of motion one obtain the first non-linear correction for lens focusing:

$$
\frac{\Delta \Phi}{\Phi}=\frac{3}{8} \frac{r r^{\prime}}{L_{\text {lens }}}
$$

For $r=1 \mathrm{~cm}, L_{\text {lens }}=15 \mathrm{~cm}$ and $r^{\prime}=1 / 15$ we obtain $\Delta \Phi / \Phi \sim 10^{-3}$. There is an additional correction related to sphericity of beryllium windows. Numerical solution for the stationary current contribution yields that this correction is about $3 \cdot 10^{-3}$.

Summarizing we can conclude that the non-linearity due to the skin effect makes the largest contribution. We will neglect other non-linearities in further calculations. As it was already mentioned maximum lens linearity is achieved at $45 \mathrm{deg}$ and this phase should be used for estimates in approximation of the linear focusing. Then for the lens gradient we can write

$$
G \approx \frac{2 I_{0}}{c r_{0}^{2}} \frac{\rho_{T i}}{\rho_{T i}+2 \rho_{L i} \frac{d_{T i}}{r_{0}}} 0.78 \approx \frac{2 I_{0}}{c r_{0}^{2}} 0.74,
$$

where $\rho_{L i}=11.4 \cdot 10^{-6} \Omega \cdot \mathrm{cm}$ and $\rho_{T i}=42 \cdot 10^{-6} \Omega \cdot \mathrm{cm}$ are resistivities for lithium and titanium, $d_{T i}$ is the thickness of titanium cylinder containing lithium, and the coefficient 0.78 is determined by field decrease due to skin effect as it is presented in Figure 15. Thus, the lens current of $500 \mathrm{kA}$ corresponds to about $74 \mathrm{kG} / \mathrm{cm}$ lens gradient. 


\section{Effect of lithium lens nonlinearity and antiproton scattering on antiproton yield}

Figure 16 presents antiproton yield as function of the lens gradient and delay time of the proton arrival relative to the time of maximum lens current. The time is measured in units of pulse $\mathrm{RF}$ phase so that $180 \mathrm{deg}$ corresponds to $350 \mu \mathrm{s}$. One can see that the maximum yield of $2.51 \cdot 10^{-5}$ is achieved at the phase of $45 \mathrm{deg}$ and the non-corrected lens focusing distance of $0.802 L_{0}$, where $L_{0}$ is the distance between target and lens, and the noncorrected lens focusing distance is computed without skin effect taken into account. As expected the value of $\mathrm{F} / \mathrm{L}$ is close to the skin effect correction of about 0.77 .

For comparison we computed the antiproton yield assuming that the magnetic field is linear and there is no multiple scattering in the lens. It yields the antiproton yield equal to $2.66 \cdot 10^{-5}$, which is about 6\% higher in comparison with the case of nonlinearity and scattering taken into account. The separation of these two effects exhibited that the lens nonlinearity actually increases the antiproton yield by $1.5 \%$, which is more probable to be related to statistical fluctuations of calculation then to a real increase. Thus observed $6 \%$ decrease in the yield is attributed to the multiple scattering in the lens. Because the effective emittance of the antiprotons coming out of the target is significantly larger of the machine acceptance the nonlinearity of the lens does not bring any harm. It pushes certain particles out of the accepted phase space but it also pulls in other particles so that the total antiproton yield is not changed.

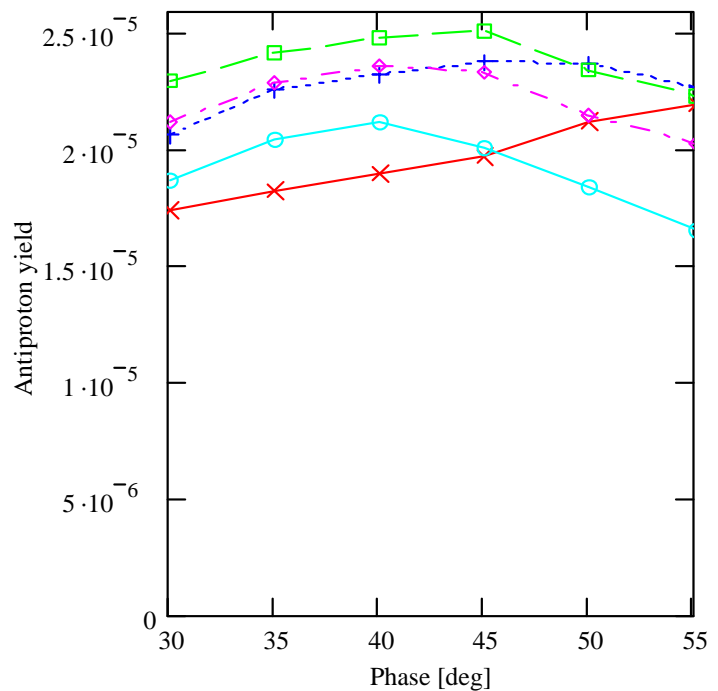

Figure 16. Antiproton yield as function of the pulse phase and the relative lithium lens focusing distance, $F / L_{0}$; the acceptance is $25 \mathrm{~mm} \cdot \mathrm{mrad}$, the target length is $8 \mathrm{~cm}$, beta-function at the target is $2.25 \mathrm{~cm}$, betafunction at the lens is $400 \mathrm{~cm}$; effective target to lens distance $-L_{0}=30 \mathrm{~cm}$.

$\times-F / L_{0}=0.734,+-F / L_{0}=0.768, \square-F / L_{0}=0.802$,

$\diamond-F / L_{0}=0.836, \circ-F / L_{0}=0.87$.

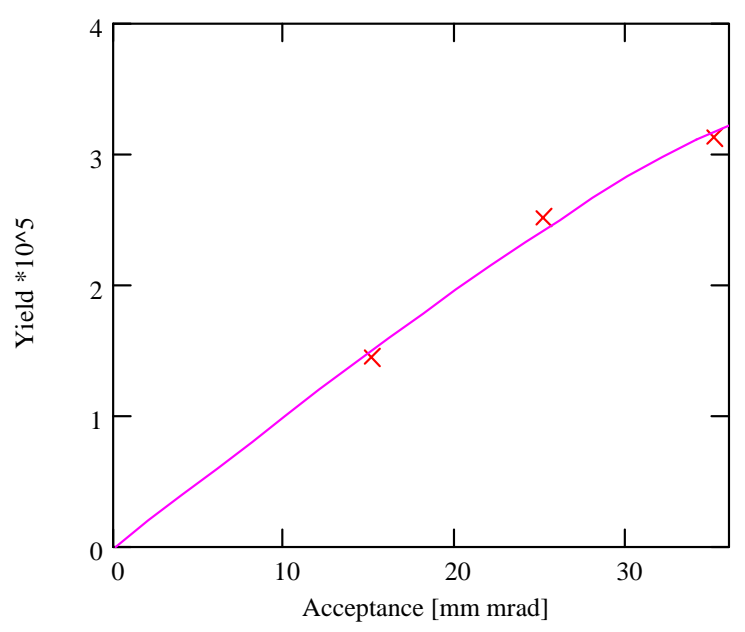

Figure 17. Computed dependence of antiproton yield on machine acceptance for currently used lithium lens with lens current of $500 \mathrm{kA}$. 


\section{Conclusions}

The historical best antiproton yield obtained at the antiproton source is equal to $1.8 \cdot 10^{-5}$. That corresponds to the acceptance of about $17 \mathrm{~mm} \cdot \mathrm{mrad}$ while the largest measured debuncher acceptance is about $25 \mathrm{~mm} \cdot \mathrm{mrad}$. It is expected that better debuncher tuning will increase the debuncher acceptance to about $35 \mathrm{~mm} \cdot \mathrm{mrad}$. Thus, improvements of optics and steering in the AP2 line and debuncher should allow an increase of antiproton yield by about 1.7 times to $3.1 \cdot 10^{-5}$ for $35 \mathrm{~mm} \cdot \mathrm{mrad}$ acceptance as shown in Figure 17.

Although the maximum lithium lens gradient, which we can reliably achieve nowadays, is significantly below the optimum we should not expect significant increase of antiproton yield with lens upgrade. To reach the maximum antiproton yield with lens of the same length $(15 \mathrm{~cm})$ one would need to increase the lens gradient by 1.4 and 1.7 times correspondingly for 25 and $35 \mathrm{~mm} \cdot \mathrm{mrad}$ acceptances. That corresponds to gradients of 105 and $127 \mathrm{kG} / \mathrm{cm}$ reaching of which is a challenging problem. And in spite of this significant increase of focusing strength that will bring only $13 \%$ and $16 \%$ antiproton yield increases corresponding to acceptances of 25 and $35 \mathrm{~mm} \cdot \mathrm{mrad}$. Minor improvement of about 3-4\% can be achieved comparatively easy by lengthening of the lens by $20-30 \%$.

Acknowledgments: The author would like to thank Peter Bussey for numerical simulation of antiproton yield from the nickel target and antiproton scattering in the lens. 